\title{
SUSTENTABILIDADE AMBIENTAL NOS SERVIÇOS DE ALIMENTAÇÃO HOSPITALAR
}

\section{ENVIROMENTAL SUSTAINABILITY IN HOSPITAL FOODSERVICES}

\author{
BÁRBARA COSTA LUDUVICE | UFS \\ ANAXÁGORA CONCEIÇÃO SOUZA|UFS \\ LAYANNE NASCIMENTO FRAGA, M.SC.|USP \\ IZABELA MARIA MONTEZANO DE CARVALHO, Dra.| UFS
}

\begin{abstract}
RESUMO
No âmbito das Unidades de Alimentação e Nutrição Hospitalares (UANSs) há produção de resíduos que são descartados durante a produção de refeições. Foi realizado um estudo transversal para levantamento das práticas de sustentabilidade de quatorze UANs hospitalares capital brasileira situadas na região Nordeste do País. Para tanto foi aplicado um questionário digital aos nutricionistas responsáveis pelas UANs, que abordou aspectos sobre gestão, controle no consumo de água, energia, prática de coleta seletiva de resíduos, descarte de óleos e gorduras, oferta de alimentos regionais com aquisição proveniente da agricultara familiar. Foi realizada análise estatística descritiva e calculadas as frequências simples e relativas, com auxílio do softwareMicrosoft Excel,versão 2013. As unidades adotam algumas práticas sustentáveis, em relação ao planejamento e elaboração do cardápio respeitando a sazonalidade dos alimentos, assim como a utilização de alimentos regionais. Contudo outras questões consideradas relevantes como as constantes capacitações dos funcionários, coleta seletiva, entre outras ainda não são realizadas nestas unidades. Diante da avaliação, observou-se que as unidades apresentam aspectos favoráveis à sustentabilidade ambiental., nNo entanto, também foram identificadas práticas inadequadas à manutenção do ambiente, assim torna-se imprescindível a reformulação das condutas nesses locais, visando à minimização dos impactos ambientais.
\end{abstract}

PALAVRAS CHAVE: Desenvolvimento sustentável; Alimentação coletiva; Gestão ambiental; Sustentabilidade.

\begin{abstract}
With in the Hospital Food and Nutrition Units (UANSs) there is waste production that is discarded during the production of meals. A cross-sectional study was conducted to survey the sustainability practices of fourteen Brazilian capital hospital UANs located in the Northeast. To this end, a digital questionnaire was applied to the nutritionists responsible for the UANs, which addressed aspects of management, control of water consumption, energy, the practice of selective waste collection, disposal of oils and fats, supply of regional food with purchase from family farmers. . Descriptive statistical analysis was performed and simple and relative frequencies were calculated, with the aid of Microsoft Excel software, version 2013. The units adopt some sustainable practices in relation to the planning and elaboration of the menu respecting the seasonality of foods, as well as the use of foods. regional However, other issues considered relevant such as the constant training of employees, selective collection, among others are not yet carried out in these units. Given the evaluation, it was observed that the units have favorable aspects for environmental sustainability, however, practices that were inappropriate to maintain the environment were also identified, so it is essential to reformulate the conducts in these places to minimize environmental impacts.
\end{abstract}

KEY WORDS: Sustainable development; Collective feeding; Environmental management; Sustainability. 


\section{INTRODUÇÃO}

A produção de resíduos e o consumo inconsciente einsustentável de produtos foram debatidos e começaram a sensibilizar várias áreas da sociedade. Nesse cenário, a Lei n 12.305, de 20101, institui no Brasil a Política Nacional de Resíduos Sólidos (PNRS), que inclui um conjunto de princípios, instrumentos, diretrizes, metas e ações que visam o gerenciamento e gerenciamento adequados dos residuos (BRASIL, 2010; PORTO, 2017; CASTRO et al., 2015). A Organização das Nações Unidas (ONU) define o termo sustentabilidade como "desenvolvimento que atenda às necessidades do presente sem comprometer a capacidade das gerações futuras de atender às suas próprias necessidades" (BARTHICHOTO; SPINELLI, 2013; VEIROS; PROENÇA, 2017; ONU, 2017).

Nesse contexto, a sustentabilidade surge como um conceito que deve ser inserido em todas as esferas que sofrem ações da atividade humana, pois em qualquer atividade, para o desenvolvimento de um produto ou serviço, podem ser gerados impactos ambientais (STRASBURG; JAHNO, 2017; SILVA et al., 2014).

Dentro destas esferas se encontra o setor de prestação de serviços de saúde, que inclui atendimento hospitalar. O número de instituições que prestam serviços de saúde vem se expandindo junto com o crescimento da população e, como outros setores de prestação de serviços, configuram-se como potenciais geradores de resíduos para o meio ambiente. Durante os cuidados hospitalares, são gerados vários tipos de resíduos, que incluem, entre outros, restos de comida e sobras produzidas durante a preparação e distribuição dos alimentos. Assim, as Unidades de Alimentação e Nutrição (UANs) localizadas nos hospitais aparecem como um elemento adicional à produção de resíduos no ambiente hospitalar (STRASBURG; JAHNO, 2017; SILVA et al., 2014; SOARES et al., 2016).

A produção de alimentos envolve uma série de etapas, desde o plantio até o preparo do produto final, envolvendo o uso de recursos ambientais para a elaboração desses processos e, consequentemente, a produção de residuos (ONU, 2017; CASTRO et al., 2014). Mesmo após o ciclo concluído do preparo dos alimentos, ainda pode haver impactos ambientais devido ao descarte das sobras, bem como as embalagens e as substâncias utilizadas para higienização dos utensílios, equipamentos e instalações. Quando descartados incorretamente, resíduos diferentes podem causar danos ao meio ambiente (RIBEIRO; JAIME; VENTURA, 2017; SANTOS, STRASBURG, 2016).

As UANs são grandes produtoras de diversos tipos de resíduos, mas os efeitos de sua liberação no meio ambiente podem ser estimulados por meio de práticas de sustentabilidade ambiental. Assim, o nutricionista responsável por uma UAN, ao gerenciar, deve observar todos os processos realizados em cada etapa da produção de alimentos, como planejamento de cardápio, aquisição de matéria-prima, armazenamento, pré-preparo e preparo, buscando estratégias para reduzir uso de recursos naturais e produção de resíduos, de forma a preservar o meio ambiente, envolvendo também treinamento de funcionários e conscientização dos usuários (ONU, 2017; RIBEIRO; JAIME; VENTURA, 2017; ABREU; SPINELLI; PINTO, 2012; MAGRINI; BASSO, 2016; POSPISCHEK, SPINELLI; MATIAS, 2014; DIAS; OLIVEIRA, 2016).

Diante do exposto, o objetivo deste trabalho foi avaliar as práticas de sustentabilidade ambiental realizadas em UANs hospitalares em uma capital da região Nordeste do Brasil, analisando todo o processo envolvido na produção de alimentos, desde a escolha de fornecedores para a aquisição de insumos, até a disposição das sobras do consumidor final.

\section{MATERIAL E MÉTODOS}

Trata-se de um estudo transversal, com base em um levantamento inicial de todas as Unidades de Alimentação e Nutrição Hospitalar da cidade de Aracaju / SE e região metropolitana, que totalizam aproximadamente 1 milhão de habitantes. Uma vez acordada a participação na pesquisa, foi aplicado um questionário digital aos nutricionistas responsáveis pelas UANs, adaptado de Martins (2017), composto por 60 perguntas para as práticas de sustentabilidade. As perguntas são divididas em 4 sessões, de acordo com os detalhes abaixo:

a) Características gerais dos restaurantes coletivos: dados sobre a operação do restaurante (modelo de gestão adotado, número de funcionários, dias de operação, número de alimentos servidos por dia e forma de distribuição dos alimentos);

b) Características dos equipamentos e instalações dos restaurantes coletivos: tipos de equipamentos, realização de manutenção preventiva e modelos das torneiras e lâmpadas predominantes no restaurante;

c) Práticas de sustentabilidade ambiental de restaurantes coletivos: presença de programas ou certificação ambiental, realização de programas contra resíduos, realização de coleta seletiva, destinação final de resíduos recicláveis, resíduos orgânicos e óleo de fritura;

d) Características específicas da sustentabilidade ambiental no processo de produção de alimentos: planejamento de cardápio, uso de alimentos regionais, alimentos sazonais, critérios para aquisição de alimentos, procedimentos para recepção e condicionamento 
de carnes, frutas e legumes, acompanhamento de quantidades per capita, procedimento de degelo, métodos de cozimento, controle do restante e sobras.

O projeto foi aprovado pelo Comitê de Ética e Pesquisa da Universidade Federal de Sergipe ( $n^{\circ} 2.412 .742$ / 2017).

As coletas ocorreram no período de agosto a dezembro de 2017 e os dados foram organizados com auxílio do software Microsoft Office Excel, versão 2013. Foram realizadas análises estatísticas descritivas e calculadas as frequências relativa e absoluta. Os serviços de alimentação foram agrupados de acordo com o sistema de gestão (autogestão ou terceirização) e as ações de sustentabilidade foram analisadas de acordo com esse parâmetro, com a ajuda do teste exato de Fisher. Foi utilizado o software Sigma Plot (versão 12.0), adotando-se o valor de $p<0,05$ para significância estatística.

\section{RESULTADOS}

Inicialmente, foram verificadas 16 instituições, enquanto a amostra final foi composta por 14 instituições hospitalares. As UANs dessas instituições são classificadas como de pequeno, médio e grande porte (SANT'ANA, 2012; HARMON; GERALD, 2007), operando diariamente por 24 horas. A tabela 1 mostra os dados do sistema de gestão, número de funcionários e alimentos produzidos nas unidades.

\begin{tabular}{|l|l|l|}
\hline Caracterização das unidades & n & $\%$ \\
\hline \multicolumn{3}{|c|}{ Sistema de gerenciamento } \\
\hline Autogestão & 8 & 57,1 \\
\hline Terceirizada & 6 & 42,9 \\
\hline \multicolumn{3}{|c|}{ No refeições servidas por dia } \\
\hline $\begin{array}{l}\text { 1 - 500 refeições } \\
\text { (pequeno porte) }\end{array}$ & 7 & 50,0 \\
\hline $\begin{array}{l}\text { 501 - 1000 refeições } \\
\text { (médio porte) }\end{array}$ & 5 & 35,71 \\
\hline $\begin{array}{l}\text { 1001 - 4000 refeições } \\
\text { (grande porte) }\end{array}$ & 1 & 7,1 \\
\hline \multicolumn{3}{|c|}{ Número de funcionários } \\
\hline 1 - 40 funcionários & 10 & 71,4 \\
\hline $\mathbf{4 1}$ - 80 funcionários & 3 & 21,4 \\
\hline 81 - 160 funcionários & 1 & 7,1 \\
\hline \multicolumn{3}{|c|}{ Forma de distribuição } \\
\hline Porcionado por funcionario & 10 & 71,4 \\
\hline $\begin{array}{l}\text { Misto (self service e porcio- } \\
\text { nado por funcionário) }\end{array}$ & 4 & 28,6 \\
\hline
\end{tabular}

Tabela 1 - Caracterização das unidades quanto, ao sistema de gerenciamento, porte, número de funcionarios e refeições produzidas por dia, Aracaju/SE, 2018.

Fonte: Autores
As práticas de sustentabilidade relacionadas aos equipamentos e instalações das unidades avaliadas estão descritas na Tabela 2.

Quando os nutricionistas foram questionados por qual motivo a manutenção preventiva do equipamento não foi realizada, foram relatados problemas em relação aos equipamentos de manutenção, como o déficit de pessoal específico para esse serviço e a manutenção apenas para fins de conserto.

\begin{tabular}{|c|c|c|}
\hline Equipamentos e instalações & $\mathbf{N}$ & $\%$ \\
\hline \multicolumn{3}{|c|}{ Número de equipamentos } \\
\hline 1 - 10 equipamentos & 2 & 14,3 \\
\hline $11-20$ equipamentos & 8 & 57,1 \\
\hline 21 - 30 equipamentos & 4 & 28,6 \\
\hline \multicolumn{3}{|c|}{ Manutenção preventiva dos equipamentos } \\
\hline Sim & 9 & 65,3 \\
\hline Não & 5 & 35,7 \\
\hline \multicolumn{3}{|c|}{ Torneiras } \\
\hline Acionamento mecânico (pedal) & 1 & 7,1 \\
\hline Temporizador (botão) & 1 & 7,1 \\
\hline Manual & 12 & 85,7 \\
\hline \multicolumn{3}{|c|}{ Iluminação } \\
\hline Fluorescente & 13 & 92,9 \\
\hline Halogênio com refletor & 1 & 7,1 \\
\hline \multicolumn{3}{|c|}{ Ar condicionado na produção } \\
\hline Sim & 1 & 7,1 \\
\hline Não & 13 & 92,9 \\
\hline
\end{tabular}

Tabela 2 - Caracterização geral dos equipamentos e instalações das Unidades de Alimentação e Nutrição hospitalares de Aracaju/SE, 2018. Fonte: Autores

As práticas gerais de sustentabilidade foram organizadas de acordo com o modelo de gestão de serviços de alimentação (autogestão ou terceirização) e são apresentadas na Tabela 3. Foram analisadas questões relacionadas a certificados, treinamento, coleta seletiva e descarte de óleo. Na presente amostra, o modelo de gestão não mostrou relação com a adoção ou não de práticas gerais para a de sustentabilidade ( $p>0,05)$.

Uma única UAN relaciona algum tipo de certificação ambiental, mas não informada. Dois nutricionistas qualificados em relação à sustentabilidade, as questões mais citadas foram o uso integral de alimentos, controle de resíduos, consumo consciente de água e energia, destino adequado de óleos e gorduras e coleta seletiva. As fontes alternativas de energia citadas foram a luz natural e o gerador de energia. As unidades que afirmaram 
desenvolver algum tipo de programa sobre desperdício de água e energia reportarão que ocorreram por meio de treinamento, orientação e alertas em locais específicos. Entre as unidades onde não havia tipo de programa, a justificativa era que a iniciativa nunca foi tomada; nunca houve cobrança pela parte administrativa; ou porque outras demandas são priorizadas .

Das instituições que relataram não realizar a coleta seletiva, foi perguntado o motivo, e foi relatado que o número restrito de funcionários dificulta a realização da coleta seletiva, bem como a falta de treinamento do equipamento para essa função. A difícil relação entre a alta administração e o nutricionista também é um fator que não contribui para a coleta, uma vez que, em alguns locais, os resíduos são separados, mas, por pertencerem a instituições maiores, não têm controle sobre destino final dos seus resíduos.

Em relação às características do cardápio, aquisição e armazenamento de matérias-primas, a Tabela 4 mostra os resultados encontrados.

Entre as instituições avaliadas, todas reportarão respeito à sazonalidade dos alimentos, bem como ao uso de alimentos regionais, durante o planejamento do cardápio. Os nutricionistas que afirmam não usar a ficha técnica de preparação relatarão que o processo de implementação estava em andamento, reivindicando a alta rotatividade no cargo como um fator limitante para a implementação deste instrumento de controle.

\begin{tabular}{|c|c|c|c|}
\hline \multirow{2}{*}{$\begin{array}{l}\text { Práticas de sustenta- } \\
\text { bilidade ambiental em } \\
\text { UANs hospitalares }\end{array}$} & \multicolumn{2}{|c|}{ Gerenciamento } & \multirow[t]{2}{*}{$p^{*}$} \\
\hline & Autogestão & Terceirizada & \\
\hline & $8(57,14 \%)$ & $6(42,86 \%)$ & \\
\hline $\begin{array}{l}\text { Certificação ambiental (a } \\
\text { unidade possui algum progra- } \\
\text { ma ou certificação ambiental) }\end{array}$ & $\operatorname{Sim} 12,50 \%$ & $\operatorname{Sim} 0,00 \%$ & 1,00 \\
\hline $\begin{array}{l}\text { Treinamento sobre susten- } \\
\text { tabilidade/ responsável (o } \\
\text { responsável recebeu algum } \\
\text { treinamento em sustentabili- } \\
\text { dade ambiental direcionado } \\
\text { a produção de refeições) }\end{array}$ & $\operatorname{Sim} 50,00 \%$ & $\operatorname{Sim} 16,66 \%$ & 1,00 \\
\hline $\begin{array}{l}\text { Treinamento sobre susten- } \\
\text { tabilidade/ funcionários } \\
\text { (funcionários da unidade } \\
\text { receberam treinamen- } \\
\text { to em sustentabilidade } \\
\text { ambiental direcionado a } \\
\text { produção de refeições) }\end{array}$ & $\operatorname{Sim} 12,50 \%$ & $\operatorname{Sim} 16,66 \%$ & 1,00 \\
\hline Fonte alternativa de energia & $\operatorname{Sim} 12,50 \%$ & $\operatorname{Sim} 16,66 \%$ & 1,00 \\
\hline $\begin{array}{l}\text { Programa Contra desperdí- } \\
\text { cio (a unidade possui algum } \\
\text { programa sobre o desper- } \\
\text { dício de água e energia) }\end{array}$ & $\operatorname{Sim} 62,50 \%$ & $\operatorname{Sim} 33,33 \%$ & 1,00 \\
\hline Coleta seletiva & $\operatorname{Sim} 62,50 \%$ & $\operatorname{Sim} 16,66 \%$ & 1,00 \\
\hline
\end{tabular}

\begin{tabular}{|l|l|l|l|}
\hline $\begin{array}{l}\text { Destino de resíduos orgâni- } \\
\text { cos (são separados e trans- } \\
\text { portados adequadamente) }\end{array}$ & $\operatorname{Sim} 50,00 \%$ & $\operatorname{Sim} 33,33 \%$ & 1,00 \\
\hline $\begin{array}{l}\text { Destino de recicláveis } \\
\text { (são separados e transpor- } \\
\text { tados adequadamente) }\end{array}$ & $\operatorname{Sim} 50,00 \%$ & $\operatorname{Sim} 66,66 \%$ & 1,00 \\
\hline $\begin{array}{l}\text { Óleo para frituras (é reali- } \\
\text { zado descarte adequado) }\end{array}$ & $\operatorname{Sim} 75,00 \%$ & $\operatorname{Sim} 66,66 \%$ & 1,00 \\
\hline *Teste exato de Fisher
\end{tabular}

Tabela 3 - Associação Tabela 3. Associação entre práticas de sustentabilidade ambiental e 0 tipo de gerenciamento UANs hospitalares do estado de Sergipe. Fonte: Autores

Durante a aquisição de alimentos, as unidades que declararem não adquirir alimentos da agricultura familiar declararão que o processo de aquisição de insumos ocorre por meio de licitações, que têm o menor preço prioritário ou que o processo de compra é realizado pela empresa-mãe e não pelo nutricionista. Em relação à alta quantitativa da não aquisição de gêneros orgânicos e agroecológicos, justificou-se que o alto valor desses produtos dificulta o processo de compra.

O recebimento dos suprimentos ocorre em $78,6 \%$ das unidades, com a realização de uma conferência do recibo de compra, inspeção das condições de entrega e do veículo, padronização da temperatura e características dos produtos, como integridade da embalagem, data de validade e selo de inspeção.

Em todas as instalações, a recepção de frutas e verduras ocorre em sua forma natural, sendo que em $78,6 \%$ dos casos esses gêneros são armazenados sob refrigeração e o restante dos alimentos armazenados em temperatura ambiente. As carnes são armazenadas sob congelamento em todas as unidades. $\mathrm{O}$ acompanhamento dos resíduos pelos clientes, da quantidade per capita de alimentos e do fator de correção alimentar, quando não realizado, foi justificado por fatores como a falta de iniciativa por parte dos nutricionistas, devido à falta de funcionários ou porque o processo estava em processo de implementação.

\begin{tabular}{|l|l|l|}
\hline Práticas Planejamento de cardápios \\
\hline \multicolumn{3}{|c|}{ n } \\
\hline Nutricionista & 13 & 92,9 \\
\hline Empresa & 1 & 7,1 \\
\hline \multicolumn{3}{|c|}{ Utilização de ficha técnica de preparação } \\
\hline Sim & 11 & 78,6 \\
\hline Não & 3 & 21,4 \\
\hline \multicolumn{3}{|c|}{ Aquisição de alimentos orgânicos } \\
\hline Sim & 4 & 28,6 \\
\hline Não & 10 & 71,4 \\
\hline
\end{tabular}




\begin{tabular}{|c|c|c|}
\hline \multicolumn{3}{|c|}{ Aquisição proveniente da agricultura familia } \\
\hline Sim & 3 & 21,4 \\
\hline Não & 11 & 78,6 \\
\hline \multicolumn{3}{|c|}{ Monitoramento per capita e fator de correção } \\
\hline Sim & 10 & 71,4 \\
\hline Não & 4 & 28,6 \\
\hline \multicolumn{3}{|c|}{ Recebimento de carnes } \\
\hline Congeladas & 11 & 78,6 \\
\hline Refrigeradas & 3 & 21,4 \\
\hline \multicolumn{3}{|c|}{ Frequência de recebimento de carnes } \\
\hline Diária & 1 & 7,1 \\
\hline Semanal & 13 & 92,9 \\
\hline \multicolumn{3}{|c|}{ Recebimento de frutas e verduras } \\
\hline Diária & 1 & 7,1 \\
\hline $2 x /$ semana & 4 & 28,6 \\
\hline $3 x /$ semana & 9 & 65,3 \\
\hline \multicolumn{3}{|c|}{ Descongelamento de alimentos } \\
\hline Temperatura ambiente & 4 & 28,6 \\
\hline Refrigeração & 9 & 65,3 \\
\hline $\begin{array}{l}\text { Temperatura contro- } \\
\text { lada (hotbox) }\end{array}$ & 1 & 7,1 \\
\hline \multicolumn{3}{|c|}{ Monitoramento desperdício } \\
\hline Sim & 7 & 50,0 \\
\hline Não & 7 & 50,0 \\
\hline \multicolumn{3}{|c|}{ Reutilização de sobras } \\
\hline Sim & 8 & 57,1 \\
\hline Não & 6 & 42,9 \\
\hline
\end{tabular}

Tabela 4 - Características específicas de sustentabilidade no processo de produção das refeições em Unidades de Alimentação e Nutrição hospitalares de Aracaju/SE, 2018. Fonte: Autores

\section{DISCUSSÃO}

As UANs avaliadas adotam pelo menos algum tipo de prática sustentável, pois foi possível observar em relação ao planejamento e elaboração dos cardápios, que respeite a sazonalidade dos gêneros, bem como o uso de alimentos regionais nesse processo. No entanto, práticas de sustentabilidade de considerável relevância, como o treinamento rotineiro dos funcionários e a coleta seletiva, ainda não são realizadas na maioria das unidades.

Grande parte das instituições avaliadas relatou realizar a manutenção preventiva do equipamento periodicamente. Esse é um fator de extrema importância para a redução dos impactos ambientais, uma vez que a manutenção adequada pode levar a uma redução de 25 a $60 \%$ no consumo de energia dos equipamentos (VEIROS; PROENÇA, 2010; HARMON; GERALD, 2007; FLÔRES; KINASZ, 2015).

As instalações das unidades avaliadas são em sua maioria torneiras de uso manual, mais suscetíveis a vazamentos e vazamentos de água e, portanto, devem conter redutores de vazão com a intenção de reduzir o consumo de água (HARMON; GERALD, 2007; FLÔRES; KINASZ, 2015).

$O$ estudo mostra que uma torneira vazando pode desperdiçar até 46 litros de água por dia, o que no final de um mês resulta em 1.380 litros de água desperdiçada, demonstrando a importância de manter as instalações hidráulicas dos estabelecimentos (SABESP, 2018). Por outro lado, a A iluminação da maioria das UANs é feita por meio de lâmpadas fluorescentes, que são eficientes, não produzem calor e permitem a distribuição adequada da luz no ambiente. Apesar disso, recomenda-se o uso máximo da iluminação natural, prática que nem sempre foi observada nas instalações investigadas (( DDIAS; OLIVEIRA, 2016; FLÔRES; KINASZ, 2015).

Entre as unidades avaliadas, 71,4\% não apresentaram nenhum tipo de certificação ambiental e, quando afirmaram possuir, não foi identificado qual era o tipo de certificação. Este documento é uma forma de garantir ao consumidor a existência de práticas sustentáveis em um determinado estabelecimento. Certificações ambientais são fornecidas a empresas ou instituições que, durante o desempenho de suas atividades, respeitam os requisitos legais de produção sustentável (ONU, 2017; SEBRAE, 2015). Essas certificações regulam e garantem que o produto ou serviço foi preparado cuidando do equilíbrio e proteção ambiental, prevenindo a poluição e as conseqüências que esses processos podem gerar sobre o meio ambiente (SEBRAE, 2015; ALONSO-ALMEIDA, 2012).

Embora quase metade das UANs (42,9\%) pratique práticas de coleta seletiva, na maioria dos casos os resíduos separados não são alocados adequadamente de acordo com o tipo de material. Os resíduos gerados por uma UAN são em geral papel, papelão, metais, vidro, equipamentos e resíduos de alimentos, sendo estes últimos gerados em maior quantidade. Sabe-se que o lixo orgânico é uma das principais fontes de impactos ambientais, uma vez que o rejunte da decomposição carrega grande quantidade de matéria orgânica, microorganismos, metais pesados e outras substâncias nocivas ao meio ambiente a curto, médio e longo prazo (CASTRO et al., 2015; SANTOS; STRASBURG, 2016). Entre as estratégias para minimizar os efeitos desses resíduos estão a redução da fonte geradora, reciclagem e compostagem e o depósito em instalações de combustível e saneamento. Entretanto, para que esses processos sejam realizados de forma eficaz, é necessário que o processo de coleta seletiva seja gerado correta e completamente (HARMON; GERALD, 2007; FLÔRES; KINASZ, 2015). Barthichoto et al. (2013) relataram que, em um total de 32 restaurantes comerciais, apenas $25 \%$ realizaram qualquer procedimento para separação de resíduos. 
Mesmo na geração de resíduos orgânicos, o óleo de cozinha é um dos elementos de maior preocupação ambiental, visto que, quando despejados inadequadamente em pias ou esgotos, podem entupir os canos, causando sérios problemas. Além disso, estudos indicam que, ao final do processo de esgotamento, quando no tratado, apenas um litro de óleo de cozinha que chega ao corpo d'água é capaz de contaminar cerca de um milhão de litros de água, prejudicando significativamente a vida marinha e fluvial, está configurada como fonte de desequilíbrio ambiental. Na maioria das unidades avaliadas, os óleos e gorduras são descartados corretamente, ou seja, coletados e destinados à produção de sabão, geralmente em associação com empresas da região, o que contribui para a economia local. (SOARES et al., 2016; CASTRO et al., 2014; RIBEIRO; JAIME; VENTURA, 2017ALONSO-ALMEIDA, 2012; MARQUES; MARQUES, 2017).

Em relação às práticas de sustentabilidade inerentes às atribuições do nutricionista, o Conselho Federal de Nutricionistas (CFN, 2005) define que, para ser considerado sustentável, o planejamento de cardápios deve cuidar da aquisição de alimentos originários da agricultura familiar, além do uso comida regional e respeito à sazonalidade dos gêneros. Tais práticas, além de proporcionar o uso de alimentos de melhor qualidade nutricional, contribuem para promover sistemas de produção mais sustentáveis e também incentivam a redistribuição de renda aos agricultores familiares (ONU, 2017; DIAS; OLIVEIRA, 2016; FLÔRES; KINASZ; MOTA et al., 2017; FERIGOLLO et al., 2017; ALAMAR et al., 2018).

Entre as UANs investigadas, a maioria $(78,6 \%)$ afirmou que não compra alimentos da agricultura familiar, uma preocupação preocupante, não um incentivo atual para a prática dessa prática. nos processos de planejamento e execução dos menus. Apesar de ter sido a maioria, ainda existe uma porcentagem considerável de locais onde essa ferramenta não é utilizada, dado que chama a atenção, uma vez que o FTP é um recurso que ajuda o nutricionista a manter o padrão, a qualidade e a qualidade. características das preparações do menu.

Além disso, esse instrumento de gestão colabora com a estimativa dos insumos quantitativos para a lista de compras e a previsão do desempenho dos preparativos. Estimase que cerca de um terço de todos os alimentos produzidos no mundo sejam perdidos ou desperdiçados, e as ações e o controle das quantidades per capita e da gordura de correção (informações contidas no FTP) aumentam a precisão da produção cardápios diários e evitar a superestimação da quantidade produzida e, consequentemente, as sobras geradas (ABREU; SPINELLI; PINTO, 2012; SOUZA; MARSI, 2016; FRANKLIN et al., 2016; SABINO; BRASILEIRO; SOUZA, 2016).
Em relação às práticas de degelo, a maioria das unidades relatou realizar esse processo sob refrigeração, sendo considerada uma prática que, quando executada corretamente, combina sustentabilidade e segurança nutricional e microbiológica, uma vez que não envolve o uso de água corrente. A literatura apresenta diferentes práticas de degelo seguro, como o uso de câmaras ou refrigeradores a 4 ${ }^{\circ} \mathrm{C}$; o uso de forno de convecção ou microondas; imersão em água com temperatura abaixo de $21^{\circ} \mathrm{C}$ por quatro horas e manutenção subsequente à temperatura ambiente, controlada localmente e livre de contaminação até a superfície atingir $3^{\circ} \mathrm{C}$; entre outros (BARTHICHOTO; MATIAS; SPINELLI, 2013; ONU, 2017).

No caso da sustentabilidade, é essencial que o nutricionista conheça seu papel no meio ambiente, participando como peça fundamental para contribuir para o desenvolvimento sustentável. Em pesquisa realizada com 193 nutricionistas, Naves e Recine (2014) identificou que as principais dificuldades na realização de práticas sustentáveis na produção de alimentos seriam falta de tempo, recursos financeiros e falta de conhecimento sobre o assunto. Esses fatores podem refletir um possível fracasso na formação profissional, bem como a escassez de especializações e cursos que abordam a sustentabilidade. Dessa forma, os nutricionistas devem procurar qualificação em relação ao assunto, e aqueles que recebem esse treinamento devem revisá-los para equipes de trabalho na forma de treinamento e treinamento constantes (MAGRINI; BASSO, 2016; FERIGOLLO et al., 2017; NAVES; RECINE, 2014).

O nutricionista é o profissional que representa o elo entre a sociedade e o sistema alimentar, uma vez que conhece as necessidades e demandas do consumidor de alimentos e todo o percurso desses alimentos em seu sistema. Assim, cabe ao nutricionista exercer apoio constante e definir estratégias para a redução da geração de resíduos, energia elétrica e água. Tais atitudes invariavelmente envolvem o treinamento das equipes envolvidas na produção de alimentos e a conscientização dos usuários dos serviços de alimentação (BARTHICHOTO; MATIAS; SPINELLI, 2013; NAVES; RECINE, 2014; REIS; FLÁVIO; GUIMARÃES, 2015).

\section{CONCLUSÃO}

O estudo mostrou que as instituições avaliadas realizam algum tipo de prática sustentável, destacando-se: uso de alimentos produzidos na região e adaptação à sazonalidade de cada alimento, uso de iluminação eficiente, descarte adequado de óleo de cozinha, utilização de dados técnicos de preparação, acompanhamento dos restos 
mortais pelos clientes e degelo de alimentos sem o uso de água corrente. Enquanto isso, com a adoção dessas práticas pela maioria das UANs, existem muitos outros comportamentos que são extremamente importantes para a sustentabilidade e devem ser implementados nessas instituições. Para tanto, é necessário que os nutricionistas responsáveis, juntamente com os gestores das instituições, busquem mais treinamentos que abordem esse tema, uma vez que ainda é pouco explorado, apesar da importância para o meio ambiente e para as gerações futuras. É necessário que a adoção dessas práticas nas unidades se torne algo rotineira e fácil de aplicar, tanto na experiência do nutricionista quanto dos colaboradores.

\section{REFERÊNCIAS}

ABREU, E.S.; SPINELLI, N.G.N.; PINTO, A.M.P. Gestão de Unidades de Alimentação e Nutrição: um modo de fazer. 2. ed. São Paulo: Metha, 2012.

ALAMAR, M.C. et al. Minimising food waste: a call for multidisciplinar research. Journal Science Food Agriculture, v. 98, n.1, p. 8-11. 2018.https://www.ncbi. nlm.nih.gov/pubmed/28963720.

ALONSO-ALMEIDA, M.M. et al. Reasos for implementing certified quality systems and impact on performance: na analysis of the hotel indutry. The Service Industries Journal, v.32, p.919-936.2012.https:// www.researchgate.net/publication/254266294_ Reasons_for_implementing_certified_quality_systems_and_impact_on_performance_An_analysis_ of_the_hotel_industry

BARTHICHOTO, M.; MATIAS, A.C.G.; SPINELLI, M.G.N. Responsabilidade ambiental: perfil das práticas de sustentabilidade desenvolvidas em unidades produtoras de refeições do bairro de Higienópolis. Revista eletrônica, v.14, n.1, p.1-12. 2013.http://revista.uepb. edu.br/index.php/qualitas/article/view/1680

BRASIL. Presidência da República. Casa Cívil. Lei no 12.305, de 2 de agosto de 2010: Institui a Política Nacional de Resíduos Sólidos. Diário Oficial da União .Brasília. 2010.

BRASIL. CFN- Conselho Federal de Nutricionistas (BR). Resolução CFN no 380/2005. Dispõe sobre a definição das áreas de atuação do nutricionista e suas atribuições, estabelece parâmetros numéricos de referência, por área de atuação, e dá outras providências. 2005.

CASTRO, R.R. et al. Management of heathcare waste in a small hospital. Revista Rene, v. 15,

p. 860-868. 2014.https://www.researchgate.net/
publication/287705035_Management_of_healthcare_waste_in_a_small_hospital

CASTRO, S.; SILVA, K.G.; SPINELLI, M.G.N.; MATIAS, A.C.G. Sustentabilidade ambiental em unidades produtoras de refeições da região central do município de São Paulo. RevistaSimbio-Logias, v.8, p. 1-13. 2015. DIAS, N.A.; OLIVEIRA, A.L. Sustentabilidade nas unidades de alimentação e nutrição: desafio para o nutricionista do século XXI. Revista de Higiene Alimentar, v.30,p. 254-255. 2016.

FERIGOLLO, D. et al. Products purchased from family farming for school meals in the cities of Rio Grande do Sul. Revista de Saúde Pública, v.51, n.6, p. 1-15. 2017. http://www.scielo.br/scielo.php?script=sci_arttext\&pid=S0034-89102017000100205

FLÔRES, T.M.; KINASZ,T.R. Cartilha para o desenvolvimento de práticas de sustentabilidade em restaurantes comerciais. Brasília. 2015.

FRANKLIN, T. et al. Segurança alimentar, nutricional e sustentabilidade no restaurante universitário. Saúde. Com,v.12, n.1, p. 482-487. 2016.http://periodicos2.uesb. br/index.php/rsc/article/view/396

HARMON, A.H.; GERALD, B.L. Position of the American Dietetic Association: food and nutrition professionals can implement practices to conserve natural resources and support ecological sustainability. Journal of the American Dietetic Association, v.107, p. 1033-1043.2007.https://www.ncbi.nlm.nih.gov/ pubmed/17571455

MAGRINI , L.N.; BASSO, C. Práticas Sustentáveis em Serviço de Alimentação Hospitalar. Ciências da Saúde , v.17, n.2, p.257-265. 2016.https://periodicos.ufn.edu. br/index.php/disciplinarumS/article/view/2034

MARQUES, E.C.; MARQUES, R.C. Roteiro para sustentabilidade na produção de refeições.Higiene alimentar, v.31, n.266, p.140-144.2017.https://pesquisa.bvsalud.org/portal/resource/pt/biblio-833501

MARTINS, A.M. Sustentabilidade ambiental em Unidades de Alimentação e Nutrição coletivas de Santa Catarina. 2017. 161f. (Dissertação de Mestrado em Nutrição). Universidade Federal de Santa Catarina, Florianópolis.

MOTA, E.B. et al. Metodologia de avaliação de cardápio sustentável para serviços de alimentação. Hollos, v.4, p. 1-14. 2017.http://www2.ifrn.edu.br/ojs/index.php/ HOLOS/article/view/5428

ONU- Organização das Nações Unidas.Report of the World Commission on Environment and Development: Our Common Future. Development 
and International Cooperation: Environment. 2017. NAVES, C.C.D.; RECINE, E. Atuação profissional do nutricionista no contexto da sustentabilidade. Demetra, v.9, n.1. p. 121-136. 2014.https://www.e-publicacoes. uerj.br/index.php/demetra/article/view/6246

PORTO, P.F. A sustentabilidade ambiental nos serviços de alimentação: o caso aplicado ao Restaurante da Universidade Federal do Maranhão. 2017. 81 p. Dissertação (Mestrado em Energia e Ambiente) Universidade Federal do Maranhão, São Luís.

POSPISCHEK, V.S.; SPINELLI, M.G.N.; MATIAS, A.C.G. Avaliação de ações de sustentabilidade ambiental em restaurantes comerciais localizados no município de São Paulo. Demetra, v.9, n.2, p. 595-611. 2014.https://www.e-publicacoes.uerj.br/index.php/demetra/ article/view/8822

REIS, H.F.;FLÁVIO, E.F.; GUIMARÃES, R.S.P. Avaliação das condições higiênico-sanitárias de uma unidade de alimentação e nutrição hospitalar de Montes Claros, MG. Revista Unimontes Científica, v.17, n.2, p.1-14. 2015.http://www.ruc.unimontes.br/index.php/ unicientifica/article/view/369

RIBEIRO, H.; JAIME, P.C.; VENTURA, D. Alimentação e sustentabilidade. Estudos Avançados, v. 31, n. 89, p.185-198. 2017.scielo.br/scielo.php?script=sci_arttext\&pid=S0103-40142017000100185

SABESP. Companhia de Saneamento Básico do Estado de São Paulo. Programa de Reciclagem de Óleo de Fritura da Sabesp. 2018.

SABINO, J.B.; BRASILEIRO, N.P.M.; SOUZA, L.T. Pesquisa de resto-ingesta em uma unidade de alimentação e nutrição hospitalar de Teófilo Otoni - MG. Higiene Alimentar, v.30, p.260-261. 2016.

SANT'ANA, H.M.P. Planejamento físico-funcional de unidades de alimentação e nutrição. (1ºd). Editora Rubio. 2012.

SANTOS, A.V.; STRASBURG, V.J. Caracterização e análise dos resíduos de embalagem gerados na produção de refeições de um hospital público de Porto Alegre, RS. Estudo e Debate em Gestão e Planejamento, v. 23, p. 296-310. 2016.http://univates.br/revistas/index. php/estudoedebate/article/view/1154

SEBRAE. Serviço Brasileiro de Apoio às Micro e Pequenas Empresas. Certificação ambiental. 2015.

SILVA, R.C. et al.Coleta e destinação final dos resíduos dos serviços de saúde hospitalar no estado do Paraná. Revista Meio Ambiente e Sustentablidade, v.7, n.3, p.644-658. 2014. https://www.uninter.com/revistameioambiente/index.php/meioAmbiente/article/ view/424

SOARES, S.G.A.et al.Responsabilidade socioambiental no contexto hospitalar: Revisão integrativa. Revista de Enfermagem, 2016; 10(11):4118-4125. 2016.https://periodicos.ufpe.br/revistas/revistaenfermagem/ article/view/11499/13366

SOUZA, L.V.; MARSI ,T.C.O. Importance of technical in UANs: production and costs preparations/meals. Health Science Institute, v.33, n. 3, p. 248-53.2016. https://pesquisa.bvsalud.org/portal/resource/pt/ biblio-2288

STRASBURG, V.J.;, JAHNO, V.D. Paradigmas das práticas de gestão ambiental no segmento de produção de refeições no Brasil. Revista de Engenharia Sanitária e Ambiental, v.22,n.2, p. 3-12. 2017.http://www.scielo. br/scielo.php?pid=S141341522017000100003\&scrip$\mathrm{t}=\mathrm{sci}$ abstract\&tlng $=\mathrm{pt}$

VEIROS, M.B.; PROENÇA, R.P.C. Princípios de sustentabilidade na produção de refeições. Nutrição e ecologia,p. 2-6.2010. 


\section{AUTORES}

ORCID: https://orcid.org/0000-0002-0234-374X

BARBARA COSTA LUDUVICE | Universidade Federal de Sergipe, Graduada em Nutrição, São Cristóvão, SE - Brasil | Endereço: Rua Dr. Osório de Araújo Ramos, 300, cond. Solar da Praia, ap. 304 - bairro 13 de Julho, Aracaju-SE, 49020700 | e-mail: barbaraluduvice26@gmail.com

ORCID: https://orcid.org/0000-0002-5247-1635

ANAXÁGORA CONCEIÇÃO DE SOUZA | Pós-graduada em nutrição Clínica e Esportiva. Universidade Tirandentes. Nutrição. Aracaju, SE - Brasil. | Endereço: Rua Existente, 189 - Santa Lúcia, Aracaju-SE, 49096-261. | e-mail: naghy.nutri@hotmail.com

ORCID: https://orcid.org/0000-0003-3208-5048

LAYANNE NASCIMENTO FRAGA, M.Sc. | Universidade Federal de Sergipe, Mestre em Ciência da Nutrição, São Cristóvão, SE - Brasil | Endereço: Avenida Corifeu de Azevedo Marques, 1284, bairro Butantã, São Paulo-SP. CEP:05339-001 | e-mail: layanne.net@hotmail.com

ORCID: https://orcid.org/0000-0002-1680-3137

IZABELA MARIA MONTEZANO DE CARVALHO, Dra. | Universidade Federal de Viçosa, Doutora em Bioquímica Agrícola, Viçosa | Endereço: Departamento de Nutrição, Avenida Marechal Rondon, S/N - Rosa Elze, São Cristóvão - SE, 49100-000 | e-mail: i.montezano.c@gmail.com

\section{COMO CITAR ESTE ARTIGO}

LUDUVICE, Bárbara Costa; SOUZA, Anaxágora Conceição; FRAGA, Layanne Nascimento; CARVALHO, Izabela Maria Montezano de. Sustentabilidade Ambiental nos Serviços de Alimentação Hospitalar. MIX Sustentável, [S.I.], v. 6, n. 3, p. 45-54, jun. 2020. ISSN 24473073. Disponível em:<http://www. nexos.ufsc.br/index.php/mixsustentavel $>$. Acesso em: dia mês. ano. doi:https://doi.org/10.29183/2447-3073. MIX2020.v6.n3.45-54. 
